\title{
Nota Farmacológica: Hiperplasia prostática benigna: actualización en su terapia farmacológica
}

Tesolin Pablo

\author{
Resumen \\ La hiperplasia prostática benigna es un problema de salud molesto y relativamente frecuente en los hombres. La siguiente nota \\ es una actualización sobre el beneficio de la evidencia existente para su tratamiento con los bloqueantes de los receptores alfa \\ o alfa bloqueantes y con los inhibidores de al 5 alfa reductasa.
}

Hiperplasia prostática benigna: actualización en su terapia farmacológica.Evid. actual. pract. ambul. 9(6);187-188 Nov-Dic 2006. Traducido con permiso y adaptado por Tesolín P de: Evidence Based Drug Therapy. Benign Prostatic Hypertrophy. An update on drug therapy. Therapeutic Letter 50. Jan. Mar. 2006.

\section{Síntesis clínica}

Los objetivos del tratamiento farmacológico de la hiperplasia prostática benigna (HPB) son la reducción de los síntomas y la prevención de las complicaciones.

Los síntomas de la HPB son clasificados como irritativos (aumento de la frecuencia miccional nocturna, quemazón, urgencia, incontinencia de urgencia) u obstructivos (dificultad al iniciar la micción, chorro débil, goteo postmiccional, vaciado incompleto de la vejiga y retención urinaria).

Tabla 1: índice sintomático de la Asociación Americana de Urología. Se suman los siete números marcados y se clasifica en: LEVE (0 a 7); MODERADA (8 a 19) y SEVERA (20 a 35).

\begin{tabular}{|c|c|c|c|c|c|c|}
\hline Preguntas a contestar & Ninguna & $\begin{array}{l}\text { Menos de una en } \\
\text { cinco veces }\end{array}$ & $\begin{array}{c}\text { Menos de la mitad } \\
\text { de las veces }\end{array}$ & $\begin{array}{c}\text { Casi la mitad de las } \\
\text { veces }\end{array}$ & $\begin{array}{l}\text { Más de la mitad de } \\
\text { las veces }\end{array}$ & Casi siempre \\
\hline $\begin{array}{l}\text { 1) Durante el último mes, } \\
\text { cuántas veces tuvo la } \\
\text { sensación de no vaciar } \\
\text { completamente su vejiga } \\
\text { luego de orinar? }\end{array}$ & 0 & 1 & 2 & 3 & 4 & 5 \\
\hline $\begin{array}{l}\text { 2) Durante el último mes, } \\
\text { cuántas veces tuvo que } \\
\text { orinar de nuevo antes de las } \\
\text { dos horas de haber } \\
\text { terminado de orinar? }\end{array}$ & 0 & 1 & 2 & 3 & 4 & 5 \\
\hline $\begin{array}{l}\text { 3) Durante el último mes, } \\
\text { cuántas veces tuvo que } \\
\text { parar y empezar de nuevo } \\
\text { mientras orinaba? }\end{array}$ & 0 & 1 & 2 & 3 & 4 & 5 \\
\hline $\begin{array}{l}\text { 4) Durante el último mes, } \\
\text { cuántas veces le resultó } \\
\text { difícil aguantar las ganas de } \\
\text { orinar? }\end{array}$ & 0 & 1 & 2 & 3 & 4 & 5 \\
\hline $\begin{array}{l}\text { 5) Durante el último mes, } \\
\text { cuántas veces tuvo un } \\
\text { chorro débil ? }\end{array}$ & 0 & 1 & 2 & 3 & 4 & 5 \\
\hline $\begin{array}{l}\text { Durante el último mes, } \\
\text { cuántas veces tuvo que } \\
\text { hacer fuerza para comenzar } \\
\text { a orinar ? }\end{array}$ & 0 & 1 & 2 & 3 & 4 & 5 \\
\hline $\begin{array}{l}\text { 7) Durante el último mes, } \\
\text { cuántas veces por noche se } \\
\text { levantó en promedio de la } \\
\text { cama para orinar desde que } \\
\text { se fue a dormir hasta que } \\
\text { se levantó por la mañana? }\end{array}$ & Nunca & Una vez & Dos veces & Tres veces & Cuatro veces & $\begin{array}{c}\text { Cinco veces } \\
\text { más }\end{array}$ \\
\hline
\end{tabular}

Las complicaciones de la HPB incluyen infecciones, retención urinaria aguda y la necesidad de realización de procedimientos.

La mayoría de los ensayos clínicos usan el Índice Sintomático de la Asociación Americana de Urología (ISAU)1 cuyo puntaje máximo es 35 puntos y que fuera desarrollado con la finalidad de de objetivar la sintomatología de los pacientes. Se describe en la tabla 1.
Cuando esta escala fue comparada con las percepciones de los pacientes, los que refirieron mejoría presentaron una reducción promedio de tres o más puntos, mientras que aquellos que no mejoraron presentaron una reducción promedio de 0,7 puntos.

\section{Bloqueantes de los receptores alfa}

En Argentina se encuentran disponibles tres bloqueantes de los receptores alfa: doxazosina, tamsulosina y terazosina.

Los alfabloquantes1 proveen un beneficio sintomático modesto. Comparados con placebo, los bloqueantes de los receptores alfa reducen los síntomas un promedio de tres puntos de la escala (ISAU).
Sin embargo, los alfa bloqueantes no previenen las complicaciones de la HPB.

La doxazosina fue comparada contra placebo durante cuatro años $(n=1493)^{2}$ no mostrando diferencias significativas en la incidencia de las complicaciones que se describen en la tabla 2.

Tabla 2: incidencia de complicaciones con doxasocina comparada

\begin{tabular}{l|c|c} 
& Doxazosina & Placebo \\
\hline Retención urinaria aguda & $1 \%$ & $2 \%$ \\
\hline Necesidad de realizar un procedimiento quirúrgico & $3 \%$ & $5 \%$ \\
\hline
\end{tabular}


con placebo.

La doxazosina se asoció a una incidencia mayor de insuficiencia cardíaca, angina de pecho y accidente cerebrovascular cuando se la comparó con clortalidona en el estudio ALLHAT, que no incluyó rama placebo. Los efectos adversos más frecuentes de doxasosina y relacionados con la dosis son mareos (incremento del riesgo frente a placebo 3-8\%) astenia (5-6\%) e hipotensión postural (3-5\%).

\section{Inhibidores de la 5 alfa reductasa: finasteride}

En un ensayo clínico a cuatro años de seguimiento ${ }^{2}$ y comparado contra placebo, el finasteride no ha demostrado una reducción significativa en el puntaje de los síntomas sintomático.

Sin embargo, un metanalisis de 16 ensayos clínicos controlados contra placebo $(n=17.456)$ con un seguimiento promedio de cuatro años demostró una reducción en la probabilidad de retención urinaria aguda y en la necesidad de efectuar procedimientos quirúrgicos, pero con considerables eventos adversos. Ver tabla 3.

Tabla 3: resultados principales del tratamiento con finasteride y sus

\begin{tabular}{l|c|c|c|c|c|c}
\hline efectos Resquitaqus SOS. & N & Finasteride & Placebo & \multicolumn{2}{|l|}{ RR (IC95\%) } & \multicolumn{2}{|l}{ RRA/RA } \\
\hline Mortalidad & 2.358 & $1,2 \%$ & $0,7 \%$ & $1,7(0,7-4,1)$ & - & - \\
\hline Eventos adversos serios & 9.180 & $11,0 \%$ & $11,4 \%$ & $0,9(0,7-1,1)$ & - & - \\
\hline Necesidad de cirugía & 15.398 & $2,1 \%$ & $4,8 \%$ & $0.6(0,4-0,8)$ & $2,7 \%$ & 37 \\
\hline Retención urinaria aguda & 14,329 & $1,1 \%$ & $3,1 \%$ & $0,4(0,3-0,6)$ & $2,0 \%$ & 50 \\
\hline $\begin{array}{l}\text { Anormalidad en la } \\
\text { eyaculación }\end{array}$ & 14.396 & $2,7 \%$ & $0,7 \%$ & $3,6(2,6-5,1)$ & $2,0 \%$ & 50 \\
\hline Disfunción eréctil & 15.839 & $8,1 \%$ & $4,8 \%$ & $1,8(1,6-2,0)$ & $3,3 \%$ & 30 \\
\hline Disminución de la libido & 14.626 & $5,3 \%$ & $3,4 \%$ & $1,7(1,4-2,0)$ & $1,9 \%$ & 53 \\
\hline
\end{tabular}

IRA: Incremento de Riesgo absoluto. NNT: Número necesario para tratar. NND: Número necesario para dañar.

En un ensayo clínico $(n=1554)$ de cuatro años de seguimiento ${ }^{5}$, cuatro $(0,3 \%)$ de los hombres desarrollaron cáncer de mama (200 veces mayor incidencia* que la esperada en esa población). En otro ensayo a siete años de seguimiento que comparó finasteride con placebo $(n=18.882)$ fue menor el numero de hombres que desarrollaron cáncer en el grupo finasteride que en el grupo placebo $(18,4 \%$ vs. $24,4 \%)$ pero en el grupo finasteride los tumores fueron de alto grado y peor pronostico, sin diferencias en la mortalidad.

\section{Comparación entre finasteride y bloqueantes de los receptores} alfa

Cinco ensayos clínicos compararon ambas drogas. No fueron encotradas diferencias en la mortalidad, en los eventos adversos serios, ni en las pérdidas del seguimiento. El grupo asignado a finasteride presentó mayor incidencia de disfunción eréctil y los pacientes asignados a alfa bloqueantes, mayor proporción de mareos, astenia e hipotensión ortostática. No hubo diferencias en la realización de procedimientos quirúrgicos $(1,6 \%$ en el grupo doxazosina vs. $2,2 \%$ en el grupo finasteride). En cuatro de los ensayos los bloqueantes de los receptores alfa redujeron en uno a tres puntos el escore de ISAU.

\section{Asociación de Finasteride y bloqueantes de los receptores alfa.}

La terapia combinada demostró una reducción mayor que el finasteride solo en resultado combinado de progresión clínica determinada por escore sintomático.

\section{Conclusiones}

- Comparados con placebo, los alfabloqueantes se asocian a una mejoría sintomática de un promedio de dos a tres puntos del escore de ISAU (35 puntos totales) no reducen las complicaciones de la HPB y se asocian a un mayor riesgo de hipotensión postural, astenia y mareos (NND: 13 a 33).

- Comparados con placebo, los inhibidores de la 5-alfa-reductasa se asocian a menor probabilidad de síntomas obstructivos (retención urinaria aguda, necesidad de procedimiento quirúrgicos (NNT: 33-50) pero empeoran la función sexual (NND: 33).

- La mayoría de los ensayos no reportaron el total de eventos adversos y la mortalidad, lo que podría tener implicancias e impacto clínico respecto del tratamiento con estas drogas.

Los pacientes deberían ser informados sobre los beneficios y las implicancias del tratamiento con estas drogas tanto en el corto como en el largo plazo.

Si bien la vida media de los alfa-bloqueantes es relativamente corta (Doxazosina $22 \mathrm{~h}$ ) es razonable comenzar con dosis bajas y evaluar la aparición de efectos colaterales a la semana, antes del ajuste de dosis. El tratamiento con finasteride no provee alivio sintomático en la mayoría de los hombres pero demostró reducir en el largo largo plazo las complicaciones de la HPB. Debe tenerse en cuenta la magnitud de sus efectos colaterales sobre la vida sexual de los pacientes.

*Ver glosario.

Recibido el 20/11/06 y aceptado 12/12/06

Referencias

1. Therapeutics Initiative, Medical management of benign prostatic hyperplasia, therapeutics setter 19(May/June 1997) http//www.ti.ibc.ca/PDF/19.PDF

2. McConnell JD, Roehrborn CG, Bautista OM, et al. The long-term effect of doxazosin, finasteride, and combination therapy on the clinical progression of benign prostatic hyperplasia. N Engl J Med 2003; 349:2387-2398.

3. Kirby RS, Roehrborn C, Boyle P, et al. Efficacy and tolerability of doxazosin and finasteride, alone or in combination, in treatment of symptomatic benign prostatic hyperplasia: the Prospective European Doxazosin and Combination Therapy (PREDICT) trial. Urology 2003;61:119-126.

4. Lepor $\mathrm{H}$; Veterans Affairs Cooperative Studies Benign Prostatic Hyperplasia Study Group. The efficacy of terazosin, finasteride, or both in benign prostatic hyperplasia.[see comment]. N Engl J Med 1996;335:533-539

5. MacDonald R, Wilt TJ. Alfuzosin for treatment of lower urinary tract symptoms compatible with benign prostatic hyperplasia: a systematic review of efficacy and adverse effects. Urology 2005; 66:780-788.

6. Lepor H; Veterans Affairs Cooperative Studies Benign Prostatic Hyperplasia Study Group. The efficacy of terazosin, finasteride, or both in benign prostatic hyperplasia.[see comment]. N Engl J Med 1996;335:533-539.

7. McConnell JD; Finasteride Long-Term Efficacy and Safety Study Group. The effect of finasteride on the risk of acute urinary retention and the need for surgical treatment among men with benign prostatic hyperplasia.[see comment]. N Engl J Med 1998;338:557-63.

8. Gormley GJ; The Finasteride Study Group. The effect of finasteride in men with benign prostatic hyperplasia. N Engl J Med 1992;327:1185-91.

9. Marberger MJ; PROWESS Study Group. Long-term effects of finasteride in patients with benign prostatic hyperplasia: a double-blind, placebo-controlled, multi-centre study. Urology 1998;51:677-86.

10. Andersen JT; The Scandinavian BPH Study Group. Can finasteride reverse the process of benign prostatic hyperplasia? A two-year placebo-controlled study. Urology 1995;46:631-7.

11. Nickel JC, Fradet Y, Boake RC, et al. Efficacy and safety of finasteride therapy for benign prostatic hyperplasia: results of a 2-year randomized controlled trial (the PROSPECT Study). CMAJ 1996; 155:1251-59.

12. Tenover JL ; Primary Care Investigator Study Group. Efficacy and tolerability of finasteride in benign prostatic hyperplasia: a primary care study. Clin Ther 1997;19:243-58.

13. Byrnes CA; CUSP Investigators. Community based study of Proscar. Efficacy, tolerability and effect on health-related quality of life of finasteride versus placebo in men with symptomatic benign prostatic hyperplasia: a community based study. Clin Ther 1995;17:956-69.

14. Beisland HO, Binkowitz B, Brekkan E et al. Scandinavian clinical study of finasteride in the treatment of benign prostatic hyperplasia. Eur Urol 1992;22:271-77.

15. The Finasteride Study Group. Finasteride (MK-906) in the treatment of benign prostatic hyperplasia. Prostate 1993:22:291-99.

16. Stoner E; The Finasteride Study Group. The clinical effects of a 5 alpha- reductase inhibitor, finasteride, on benign prostatic hyperplasia. J Urol 1992;147:1298-1302.

17. Lukkarinen O, Lehtonen T, Talja M, et al. Finasteride following baloon dilatation of the prostate. A double-blind, placebo-controlled, multicentre study. Ann Chir Gynaecol 1999;88:299-303.

18. Kirby RS, Bryan J, Eardley I, et al. Finasteride in the treatment of benign prostatic hyperplasia. A urodynamic evaluation. Br J Urol 1992;70:65-72

19. Yu HJ, Chiu TY, Lai MK. Therapeutic effects of finasteride in benign prostatic hyperplasia: a randomized double-blind controlled trial. J Formos Med Assoc 1995;94:37-41

188 EVIDENCIA - Actualización en la Práctica Ambulatoria - Noviembre/Diciembre 2006 - Disponible en internet: www.evidencia.org

volumen 9 nro. 6 